\title{
SUMMARY OF ARTICLES
}

repeated the experiments of Chesney, which showed that after intravenous inoculation with $S$. pallida these organisms settled particularly in the scars following wounds previously produced in the back.

In each of four rabbits they produced on the right side of the back three wounds each about $\mathrm{I} \cdot 2 \mathrm{~cm}$. in diameter, designated $\mathrm{A}, \mathrm{B}$, and $\mathrm{C}$, respectively, from the head towards the tail, and on the left side three similar wounds, $\mathrm{A}^{\mathbf{1}}, \mathrm{B}^{\mathbf{1}}$, and $\mathrm{C}^{\mathbf{1}}$ (in order) from tail towards head, wounds $\mathrm{A}$ and $\mathrm{A}^{1}$ being made first, $\mathrm{B}$ and $\mathrm{B}^{1}$ a week later, and $\mathrm{C}$ and $\mathrm{C}^{1}$ a week after that. Three weeks after production of $\mathrm{C}$ and $\mathrm{C}^{1}$ all four animals were inoculated intravenously with $5^{\circ}$ o c.c. of an emulsion of $S$. pallida. In all four animals papules developed in all scars, and they were most pronounced in the freshest ones, $\mathrm{C}$ and $\mathrm{C}^{1}$. The experiments thus confirm those of Chesney in showing the importance of trauma in determining the sites of syphilitic lesions.

\section{W. HARRISON.}

(I) ANNUAL REPORT OF THE CITY AND ROYAL BURGH OF EDINBURGH V.D. SCHEME. BY DR. R. C. L. Batchelor, Acting Clinical Medical Officer.

(2) ANNUAL REPORT ON THE V.D. SCHEME OF THE CITY OF SALFORD. By Dr. E. Tytler Burke.

THEse two reports are for the year I934, and their simultaneous arrival enables interesting comparisons to be made between two of the best-known venereal diseases schemes outside London.

The first point to strike the reader is that the Edinburgh report deals with the whole of the venereal diseases work administered by the Corporation, inasmuch as the Venereal Diseases Officer controls not only the Venereal Diseases Clinic at the Royal Infirmary, but also the venereal diseases treatment at the Hospital for Women and Children and its subsidiary centres, that done at the Royal Maternity Hospital and the Seamen's Dispensary, Leith, and the care of venereal diseases in-patients in the Municipal Hospitals. The Edinburgh report also devotes a section to ophthalmia neonatorum; and, presumably, therefore, the venereal diseases aspects of these 


\section{BRITISH JOURNAL OF VENEREAL DISEASES}

patients (and their mothers) are also dealt with by Dr. Batchelor.

The Salford report, on the other hand, relates only to the venereal diseases clinic and its associated beds. Another major point of difference is the great difference in the amount of in-patient treatment provided. In Edinburgh, 296 men and I36 women received in-patient treatment at the Royal Infirmary-these numbers are apart from in-patients dealt with under the Scheme at the Municipal, Bruntsfield, Elsie Inglis and the Royal Maternity Hospitals. In Salford, only 3 men and 43 women were admitted for in-patient treatment under the Scheme. These are out of 2,725 new patients in Edinburgh and I,97I in Salford. In Edinburgh, however, 709 of the new patients were found not to be suffering from venereal disease, and in Salford the number was 909.

There were 32I new male syphilis patients in Edinburgh and 79 patients (presumably both new and old) were admitted to hospital. There were I58 new male cases of syphilis in Salford (approximately half the Edinburgh number) and there was only one admission to hospital. Male in-patient treatment in Edinburgh appears to be confined to the Royal Infirmary, and no figures are given in the Salford report as to the cases of venereal diseases treated as in-patients in the Municipal Hospital, but it is quite clear that the criteria used for determining whether in-patient treatment for male cases of syphilis is to be provided or not cannot be the same in these two cities.

The lay-out and form of presentation of the reports are quite different. In the Salford report the standard form of return required by the Ministry of Health for England and Wales is included: in the corresponding section for Edinburgh the figures can only be obtained, and only in part, by consulting tables compiled on a different basis. In the Edinburgh report a feature is made of the monthly incidence of the work done. Apart from the features already mentioned the chief points for comment in the Edinburgh report are :-

(I) Of the 2,803 new patients dealt with under the Scheme at all the centres and "found to be suffering from venereal infection," 705, or $25 \cdot \mathrm{I}$ per cent., were suffering from " non-specific V.D.," which is stated to include such conditions as " non-gonorrhœal urethritis, 


\section{REPORTS ON V.D. SCHEMES}

balanitis and non-syphilitic ulceration." It is probable that in other clinics these would be returned as "nonV.D." and not as " non-specific venereal disease." In the Royal Infirmary Clinic out of 2,725 new patients, 5I5 were " non-specific V.D." and 709 were returned as "no V.D."

The Salford figures are I,97I new cases, of which 909 were " non-V.D." These 909 " non-V.D." are 46 per cent. of the total new cases, and the $5 \mathrm{I}_{5}$ "non-specific V.D." and the 709 " no V.D." in Edinburgh, when added together, are 45 per cent. of the new cases. This suggests that " non-V.D." in Salford includes the Edinburgh group of " non-specific V.D."

(2) At the Royal Infirmary out-patient attendances numbered 87,789 male and 18,842 female. The Salford figures are 72,648 male and 23,858 female attendances.

(3) There is a section on vulvo-vaginitis, and reference is made to the great havoc which it can cause in a children's hospital or ward.

(4) Stabismol, which is referred to in Salford as being used as part of the standard treatment, is stated in Edinburgh not to have been found to differ materially in efficiency from the older suspensions of bismuth metal.

(5) Under the excellent Edinburgh "follow-up" system, 2,4II visits were paid by the nurse-almoner to women and children defaulters.

Dr. Batchelor is continuing the traditions set up by Dr. Lees and presents a record of an admirable year's work.

The Salford report is twice as big as the Edinburgh one, and is presented in a most attractive style. It contains a number of valuable diagrams and tables.

The main impression obtained from the Report is the superiority of the present ad hoc clinic established in I928 over its predecessor. Comparing the I934 figures with those for I927 the patients increased by 243 per cent., the attendances by 5I9 per cent., serological tests by 2,6 I3 per cent. and the cure-rate by 300 per cent., while the defaulter rate declined by 76 per cent. - a magnificent record.

The main points are :-

(I) In I934 there were 652 " fresh infections" in men compared with 87 " old infections." In women there were I68 "fresh" and 53 " old" infections. "Fresh 


\section{BRITISH JOURNAL OF VENEREAL DISEASES}

infections " are defined as those in which the disease is less than twelve months old.

(2) The proportion of male gonorrhœa to male syphilis cases is 5.7 to I (" fresh infections ").

(3) The proportion of men patients to women is about 4 to $\mathrm{I}$.

(4) Intravenous injections for syphilis are given thrice weekly.

(5) The "dangerous" defaulter rate appears to be I2.6 per cent. Prior to the establishment of the clinic in its present situation, the ratio of defaulters was greater than that of cured cases.

(6) The cost of the clinic was $£ 9,675$ and the cost per attendance $2 \cdot 02 s$.

(7) A new classification of syphilis is given in lieu of the old Ricordian terms, primary, secondary, tertiary, etc.

(8) The full scheme of treatment used is set out in detail.

(9) The course of treatment for congenital syphilis lasts five years " irrespective of what the serology may be at any time during that period."

(I0) An interesting section on compulsory treatment is given :-

"Voting papers were issued to patients, and they were asked to answer 'Yes' or 'No' to the following questions :-

"Question No. I. Should a person suffering from a venereal disease be compelled to receive treatment, provided it is given confidentially and free?

"Question No. 2. Should the parents or guardians of children suffering from venereal disease be compelled to have such children treated, provided it is confidential and free?

" The results were :-

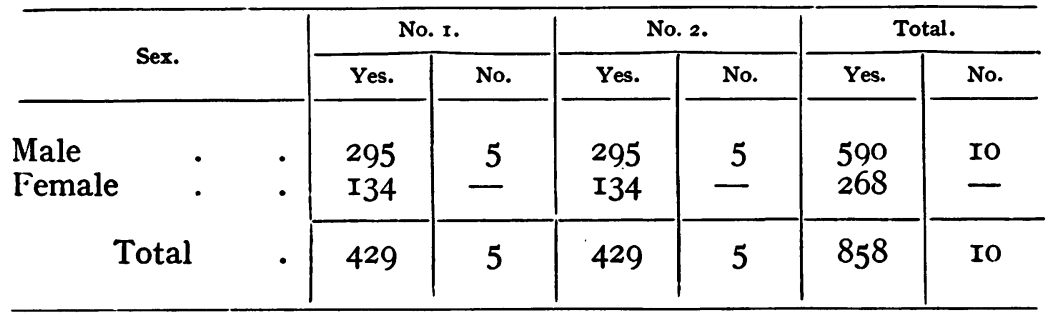




\section{REPORTS ON V.D. SCHEMES}

" In other words, the opinion among patients is overwhelmingly in favour of compulsion being exercised on the defaulter."

(II) Reference is made to the dangers of neurosyphilis in drivers of vehicles and to the "lorry girls."

The Salford clinic is obviously very much alive and is a great credit to Colonel Burke and all who are associated with it.

W. A. D. 